\title{
Letter
}

\section{In reply to Dr RG Pringle}

The letter of Dr RG Pringle raises several problems:

(1) The first point is the distinction made by Dr Pringle between, on the one hand Cyst and Syrinx which are neuroradiological findings and, on the other hand Posttraumatic Syringomyelia (PTS) which is considered as a progressive clinical disorder. We do not agree with this distinction: cysts are stabilised lesions confined to the site of injury, whilst syrinxes are progressive and extend beyond the limit of the vertebral injury and the initial extent of the lesion-syndrome. This is what our own experience has taught us, and has previously been demonstrated in the literature. ${ }^{1}$ From a pathophysiological point of view, syrinxes and post-traumatic syringomyelia (PTS) are the same phenomenon, the PTS being the clinical expression of extended syrinxes. The neuroradiological distinction between cysts and syrinxes is not artificial and is easily demonstrated on good MRI images.

(2) Dr Pringle does not agree with our sentence: 'the incidence of PTS is increasing in the recent literature'. This results from a misunderstanding, what is said is that the incidence of PTS is increasingly recognised, partially because of the development of spinal injury treatment centers and the systematic follow-up of this population by specialized teams, and in part by the improvement of the neuroradiological means of investigation (ie MRI).

(3) Our incidence of neurological change is higher than the incidence found by Wang in 1996 (23\%). These neurological changes include clinical symptoms which are subjective ones such as neurogenic pain, increased spasticity, hyperhydrosis, and clinical signs such as ascending sensory level, loss of reflexes, motor deficit. In half of the patients we found no syrinxes but in $36 \%$ of these cases, we found atrophy. This observation raises the problem of post-traumatic non cystic myelopathy. The explanation of this high incidence of neurological change can be due to the fact, that in all patients reviewed we systematically sought for symptoms and signs of PTS. Dr Pringle evokes a possible bias of selection. If it exists, it is minor because we follow systematically our patients, yearly in $80 \%$ of the cases. We cannot totally eliminate the fact that patients with problems are more compliant with follow up and with investigations.

To conclude, the objective of this study was to correlate the occurrence of syrinxes with canal stenosis. There is no confusion in our mind between neuroradiological sequelae of the injury as cysts, and syrinxes and PTS which are the same pathophysiological process.

Dr B Perrouin-Verbe

\section{References}

1 Wang D et al. A clinical magnetic resonance imaging study of the traumatised spinal cord more than 20 years following injury. Paraplegia. 1996; 34: 65-81. 